\title{
A NO-REFERENCE PERCEPTUAL BLUR METRIC
}

\author{
Pina Marziliano, Frederic Dufaux, Stefan Winkler and Touradj Ebrahimi* \\ Genimedia SA, Grand-Chêne 8 \\ 1003 Lausanne, Switzerland \\ \{pmarziliano,fdufaux, swinkler,tebrahimi\}@ genimedia.com
}

\begin{abstract}
In this paper, we present a no-reference blur metric for images and video. The blur metric is based on the analysis of the spread of the edges in an image. Its perceptual significance is validated through subjective experiments. The novel metric is near real-time, has low computational complexity and is shown to perform well over a range of image content. Potential applications include optimization of source coding, network resource management and autofocus of an image capturing device.
\end{abstract}

\section{INTRODUCTION}

The perceptual quality of digital images or digital video is very important today as consumers are getting increasingly educated with these technologies and thus are more demanding. In order to improve the perceptual quality of a digital image or video one must identify and measure, objectively and subjectively, the different perceptual artifacts that come about. Many perceptual artifacts exist, for example, blockiness in MPEG compression, or blurriness and ringing in JPEG2000 compression [5].

Most existing metrics require a reference together with the processed image or video in order to evaluate the visibility of these artifacts [7]. This imposes obvious limitations on the applications that such a metric can be used for. Noreference metrics are much more powerful. No-reference signifies that the metric is not relative to the original but is an absolute value associated to a given image or video sequence. A number of such metrics have already been developed for blockiness, for example [6]. Much less attention has been devoted to other impairments so far.

Measuring the perceptual blur in an image or a video sequence has not yet been investigated. Related research includes blur identification [4], blur estimation [2], image deblurring [1] and blind deconvolution [3]. Both deblurring and blind deconvolution can be used to recover the original image and therefore a full-reference metric becomes appli-

\footnotetext{
* Prof. T. Ebrahimi is also with the Signal Processing Institute at the Swiss Federal Institute of Technology Lausanne, Switzerland.
}

cable. In practice these require iterative solving methods which are computationally demanding.

In this paper, we are mainly concerned with blur estimation. We aim at a general blur measurement technique without making any assumptions on the blurring process. We first give a brief definition of blur, then we introduce our content-independent no-reference perceptual blur metric. The perceptual blur measurement is defined in the spatial domain as the spread of the edges. This metric is of low computational complexity. Finally we show via subjective experiments that this novel perceptual blur metric is highly correlated to the subjective blur ratings.

\section{NO-REFERENCE BLUR METRIC}

An image appears blurred when its high spatial frequency values in the spectrum are attenuated. Different types of blurs exist. For example, motion blur due to the relative motion between the camera and the scene, and out of focus blur due to a defocused camera and lens aberrations [4]. Blur can also be introduced when processing the image data, such as performing compression.

In this section, we propose a no-reference blur measurement technique. We assume no knowledge of the original image, and do not make any assumptions on the type of content or the blurring process. The result is an objective measure which correlates with the perception of blur.

The blur measurement is defined in the spatial domain. Blur is perceptually apparent along edges or in textured areas. Our technique is based on the smoothing effect of blur on edges, and consequently attempts to measure the spread of the edges. In practice, we have observed that it is sufficient to measure blur along vertical edges.

The algorithm is summarized in Fig. 1. First we apply an edge detector (e.g. vertical Sobel filter) in order to find vertical edges in the image. We then scan each row of the image $^{1}$. For pixels corresponding to an edge location, the start and end positions of the edge are defined as the local extrema locations closest to the edge. The edge width is

\footnotetext{
${ }^{1}$ The method can easily be extended to horizontal edges by filtering with a horizontal Sobel filter and then scanning each column.
} 


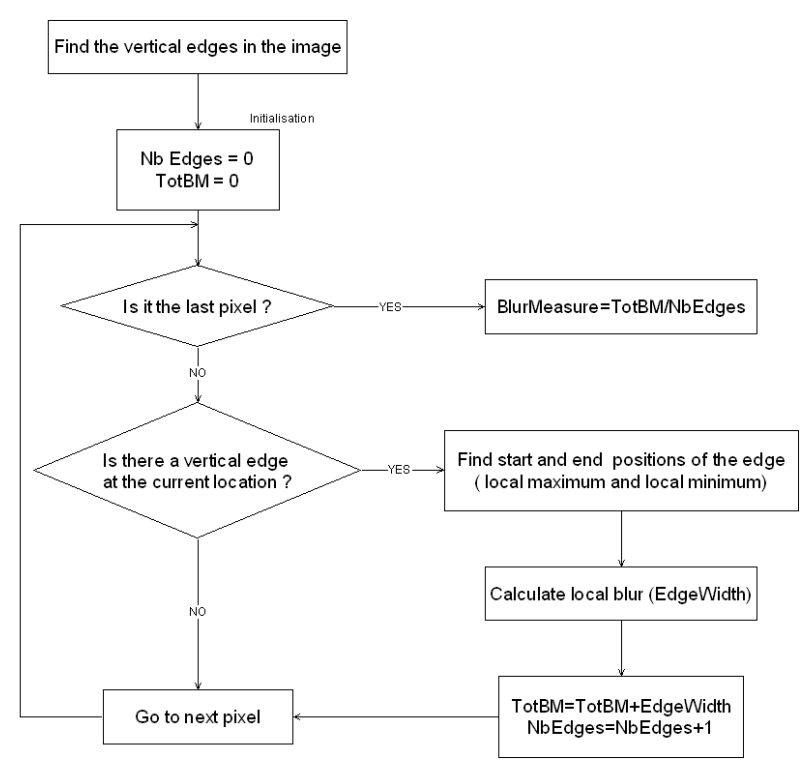

Fig. 1. Flow chart for no-reference edge-based blur measurement. TotBM denotes the total blur measurement, NbEdges denotes the number of edges.

then given by the difference between the end and start positions, and is identified as the local blur measure for this edge location. Finally, the global blur measure for the whole image is obtained by averaging the local blur values over all edge locations.

An example of a row in a image is illustrated in Fig. 2. For the edge location $P 1$, the local maximum $P 2$ defines the start position, while the local minimum $P 2^{\prime}$ corresponds to the end position. The edge width is $P 2^{\prime}-P 2$ or 11 pixels for this example. Similarly, for the edge $P 3$, the local minimum $P 4$ is the start position, the local maximum $P 4^{\prime}$ is the end position, and $P 4^{\prime}-P 4$ is the edge width.

For color images, blur is measured on the luminance component Y. While the above description considers still images, it is straightforward to extend the technique to digital video by measuring blur in every frame.

\section{EXPERIMENTS AND RESULTS}

Here, we show results for the no-reference edge-based blur measurement defined in the previous section. We also describe a subjective experiment and compare the results between the subjective and objective blur measurements.

In the following experiments we consider five color test images of size $768 \times 512$ and 24 bits $R G B$ as shown in Fig. 3 and Fig. 5(a). We examine two types of blur. The first set of blurred images is obtained by filtering the five original images with a Gaussian filter with standard deviations $\sigma \in$ $\{0.4,0.8,1.2,1.6,2\}$ pixels. The second set of blurred im-

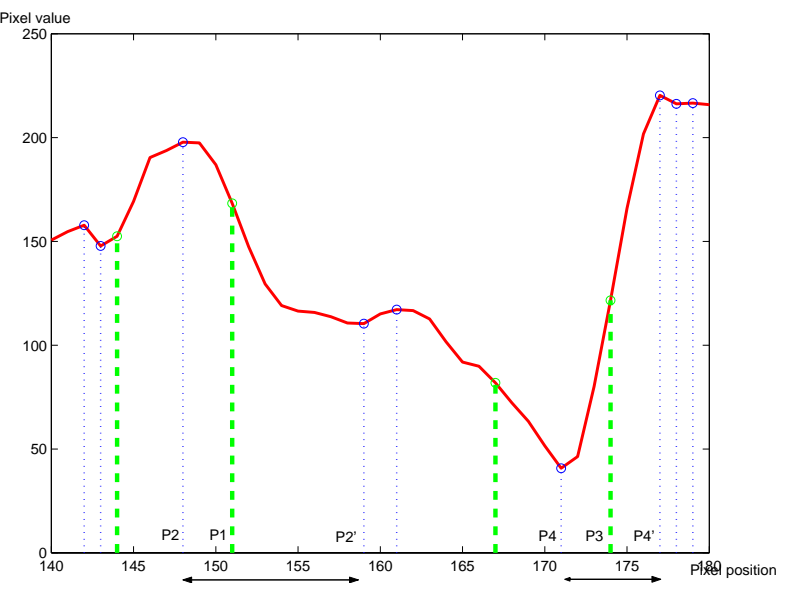

Fig. 2. One row of the blurred image. The detected edges are indicated by the dashed lines, and local minima and maxima around the edge by dotted lines. The edge width at $P 1$ is $P 2^{\prime}-P 2$.

ages is obtained using the JPEG 2000 compression scheme with compression ratios $C_{R} \in\{40,80,120,160,200\}$. Figure 4

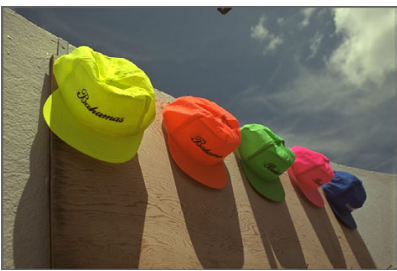

(a)

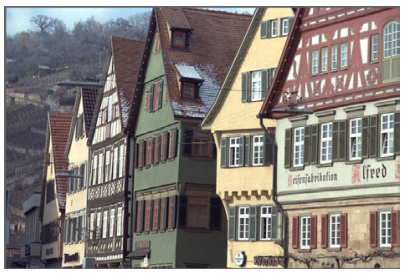

(c)

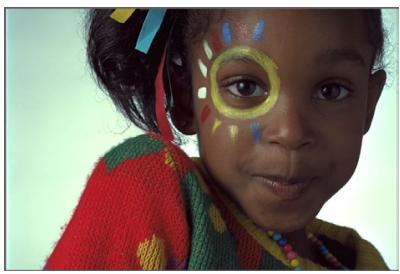

(b)

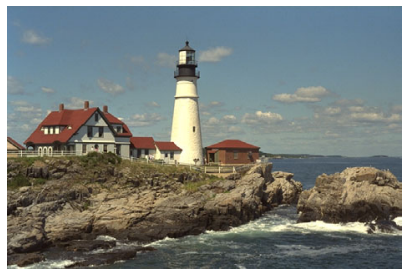

(d)
Fig. 3. Test images: (a) caps; (b) girl; (c) houses; (d) lighthouse.

illustrates the behavior of the no-reference edge-based blur metric at each distorsion level. This strong linear relation is consistent for all the test images.

We validate our perceptual blur metric by executing a subjective test on a set of 55 images composed of the 5 original images plus 25 Gaussian blurred images ( $=5$ test images $\times 5$ levels of distortion) plus 25 JPEG 2000 compressed images. The subjective test setup is as follows: Ten expert viewers were asked to quantify on a scale of 0 to 10 , (where zero is no blur and ten is lots of blur, see Fig. 5), the amount 


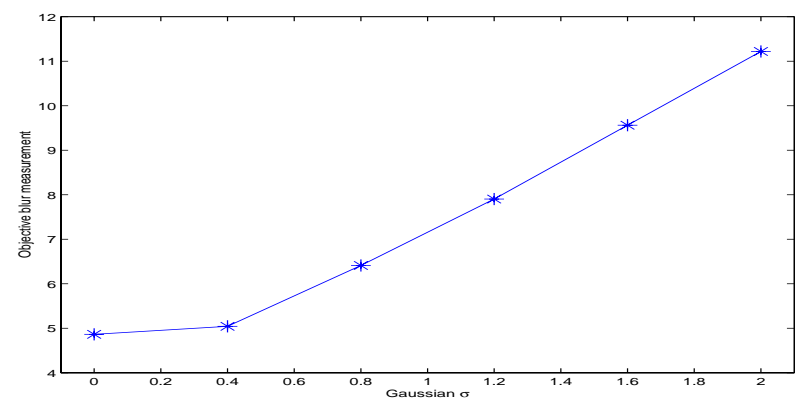

(a)

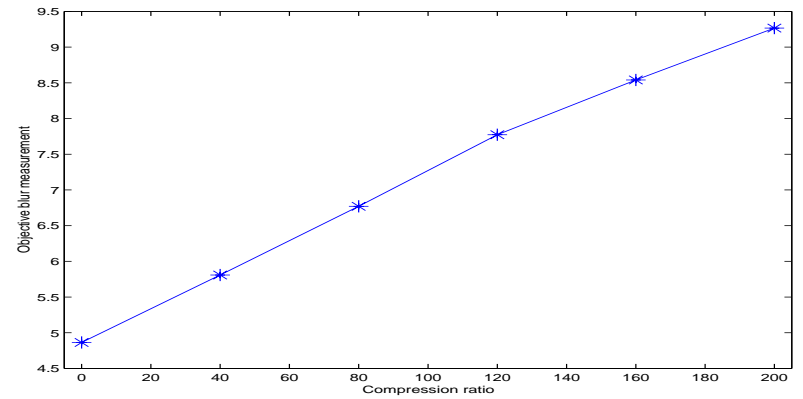

(b)

Fig. 4. Behavior of no-reference edge-based blur metric for the motocross test image. (a) Objective blur measurement versus the standard deviation, $\sigma \in\{0.4,0.8,1.2,1.6,2\}$ of the Gaussian blurring filter employed to blur the image; (b) Objective blur measurement versus JPEG 2000 compression ratio $C_{R} \in\{40,80,120,160,200\}$.

\begin{tabular}{|c|c|c|}
\hline & Linear & Spearman's rank order \\
\hline Gaussian & $96 \%$ & $96 \%$ \\
\hline JPEG 2000 & $85 \%$ & $87 \%$ \\
\hline
\end{tabular}

Table 1. Correlation between subjective testing and our perceptual blur metric.

of blur in these images.

The subjective blur rating is simply the average of the ten votes. Figure 6 illustrates the high correlation between the subjective blur ratings and our no-reference edge-based blur metric. The $95 \%$ confidence intervals are also illustrated in the error bar plots. The correlation values are summarized in Table 1. The expert viewers found it more difficult to distinguish blur from the ringing artifact in the JPEG 2000 compressed images, which explains for the lower correlation, with respect to Gaussian blurred images.

\section{CONCLUSION}

We defined a no-reference edge-based blur metric. The novel metric shows a high correlation with the subjective ratings.

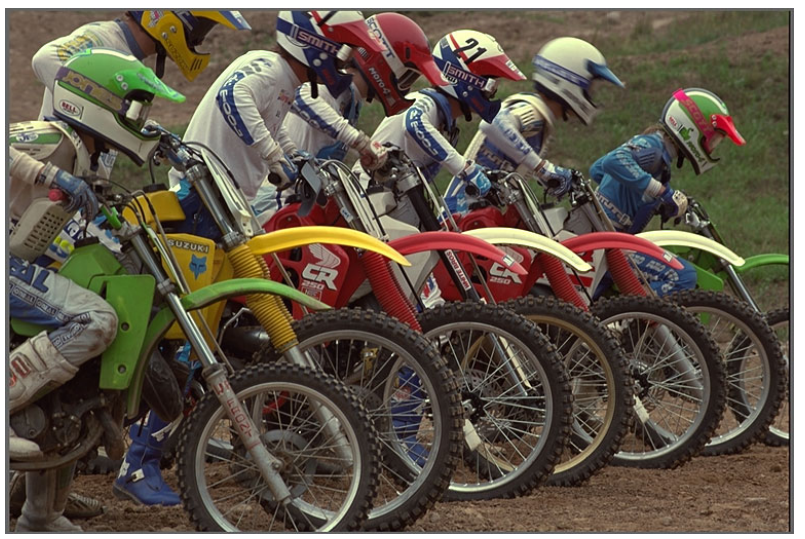

(a)

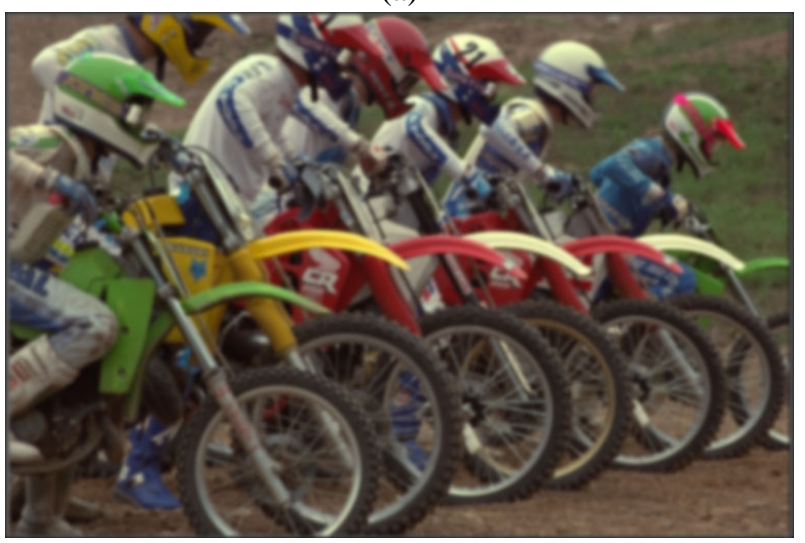

(b)

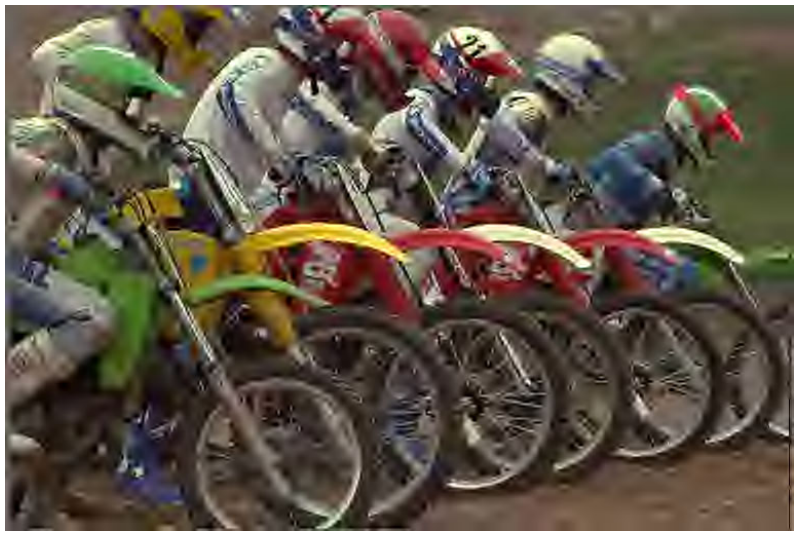

(c)

Fig. 5. Motocross test image with (a) no blur; (b) lots of blur in Gaussian blurred image; (c) lots of blur in JPEG2000 compressed image. 
It is near real-time, has low computational complexity and its performance is independent of the image content. Applications of this metric involve source coding optimization, network resource management and autofocusing of a capturing device. Future research includes the measurement of other type of artifacts such as ringing.

\section{REFERENCES}

[1] A. S. Carasso. Linear and nonlinear image deblurring: A documented study. SIAM Journal on Numerical Analysis, 36(6):1659-1689, 1999.

[2] J. H. Elder and S. W. Zucker. Local scale control for edge detection and blur estimation. IEEE Trans. on Pattern Analysis and Machine Intelligence, 20(7):699716, July 1998.

[3] D. Kundur and D. Hatzinakos. Blind image deconvolution. IEEE Signal Processing Magazine, 13:43-64, May 1996.

[4] R. L. Lagendijk and J. Biemond. Basic Methods for Image Restoration and Identification, chapter 3.5, pages 125-139. Academic Press, 2000.

[5] D. S. Taubman and M. W. Marcellin. JPEG2000: Image Compression Fundamentals, Standards and Practice. Kluwer Academic Publishers, 2002.

[6] Zhou Wang, Alan C. Bovik, and Brian L. Evans. Blind measurement of blocking artifacts in images. In IEEE Proc. ICIP, volume 3, pages 981-984, Vancouver, Canada, September 10-13, 2000.

[7] Stefan Winkler. Vision Models and Quality Metrics for Image Processing Applications. $\mathrm{PhD}$ thesis, École Polytechnique Fédérale de Lausanne, Switzerland, 2000.

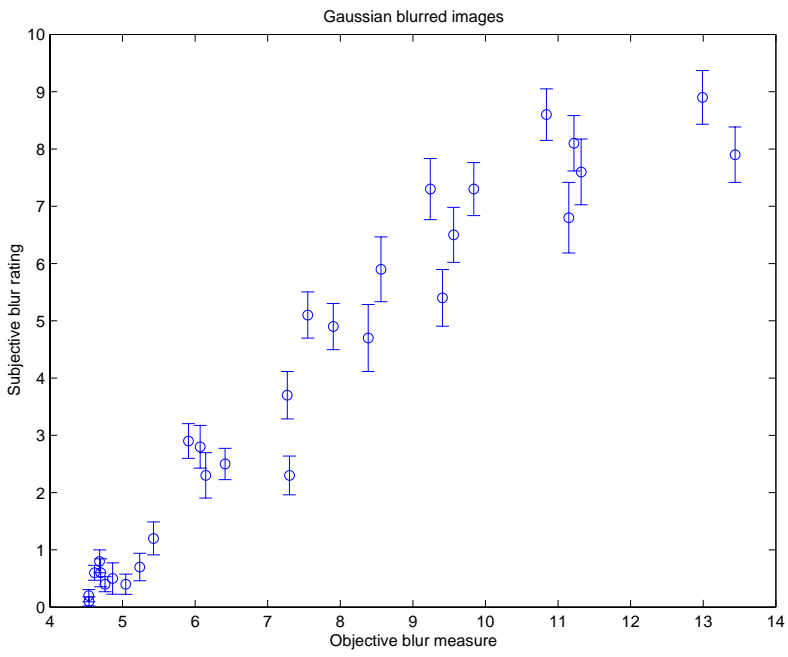

(a)

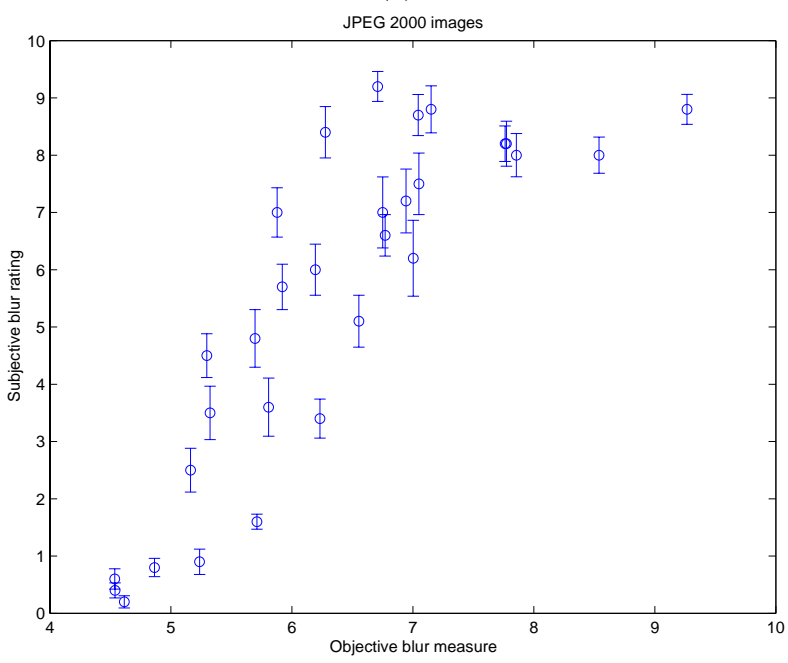

(b)

Fig. 6. Error-bar plot with $95 \%$ confidence intervals of subjective blur ratings versus the objective no-reference edgebased blur measurement. (a) Gaussian blurred images; (b) JPEG2000 compressed images. 